\title{
LIDAR STUDY OF WIND TURBULENCE, LOW LEVEL JET STREAMS, AND ATMOSPHERIC INTERNAL WAVES IN THE BOUNDARY LAYER OF ATMOSPHERE

\author{
Viktor Banakh $^{1 *}$, Igor Smalikho ${ }^{2}$
} \\ ${ }^{1,2}$ Institute of Atmospheric Optics of Siberian Branch of the Russian Academy of Sciences, Russia, *banakh@iao.ru
}

\begin{abstract}
The results of lidar study of wind turbulence, low level jet streams, and internal atmospheric waves in the stable boundary layer of atmosphere on the coast of Lake Baikal are presented. Few events of the atmospheric internal waves (AIWs) were registered during the experimental campaign. All the registered AIWs were observed in the presence of low level jet streams. Two dimensional time-height patterns of the wind turbulence dissipation rate during AIW events were obtained as well.
\end{abstract}

\section{INTRODUCTION}

Wind turbulence, low level jet streams, and atmospheric internal waves (AIWs) were studied on the coast of the Lake Baikal in summer campaigns of 2015 and 2016 with the use of a Stream Line lidar (Halo Photonics). The lidar was mounted $340 \mathrm{~m}$ away from the coast of the lake, on the territory of the Baikal Astrophysical Observatory of Institute of Solar-Terrestrial Physics, Siberian Branch, Russian Academy of Sciences $\left(51^{\circ} 50^{\prime} 47.17^{\prime \prime N}\right.$, $104^{\circ} 53$ '31.21"E); the lidar altitude above the lake level was $180 \mathrm{~m}$. The analysis of the lidar data processing results shows several AIW events occurred in the presence of jet streams. Two jet streams were observed simultaneously during some AIW events. Using the initial data of these measurements (arrays of radial velocities and signalto-noise ratios (SNR)) we also retrieved the timeheight patterns of the turbulence kinetic energy dissipation rate and calculated the relative error of assessment of the dissipation rate from the lidar data.

\section{METHODOLOGY}

Stream Line lidar measurements were carried out under the following parameters: elevation angle $\varphi=60^{\circ}$; number of pulses used for data accumulation $N_{a}=3000 ;$ angular speed of probing beam scanning $\omega_{c}=10 \%$ s; measurement time per scanning of $36 \mathrm{~s}$; resolution in the azimuth angle $\Delta \theta=2^{\circ}$, and the number of rays per scanning $M=180$.

To retrieve the vertical profiles of the wind speed $U$, the wind direction angle $\theta_{V}$, and the vertical component of the wind velocity $V_{z}$, the method of filtered sine wave fitting was used [1, 2]. This method allows estimation of the mean wind velocity with acceptable accuracy from very noisy arrays of radial velocities $\hat{V}_{r}\left(R_{k}, \theta_{m}, n\right)$ containing up to $50 \%$ of bad estimates $\hat{V}_{r}$. Here $R_{k}=R_{0}+k \delta R$ is the distance, $R_{0}$ is the minimal sounding range, $k=0,1,2, \ldots, K$, $\delta R=3 \mathrm{~m}, \theta_{m}=m \Delta \theta$ is the azimuth angle, $m=1,2,3, \ldots, M, n$ is the number of current scan. As shown in [3] for $N_{a}=3000$, the minimal signal to noise ratio is $0.005(-23 \mathrm{~dB})$, under which the wind speed estimation error does not exceed $0.1 \mathrm{~m} / \mathrm{s}$.

The turbulence kinetic energy dissipation rate $\varepsilon$ is one of key parameters of the wind turbulence. The turbulence structure in its inertial interval is completely determined by this parameter and obeys the Kolmogorov law [4]. To assess the turbulence energy dissipation rate $\varepsilon$, we used the method of transverse (azimuth) structure function of the radial velocity measured by the lidar during conical scanning [5]. Therefore, a high resolution in the azimuth angle was important during the measurements. At the same time, the tangential speed of the volume sounded movement $V_{t}=\omega_{c} R_{k} \cos \varphi$ should be much more than the mean wind speed at the measurement altitude. For 
correct estimate of the dissipation rate $\varepsilon$ from the array $\hat{V}_{r}\left(R_{k}, \theta_{m}, n\right)$, the probability of bad estimation of the radial velocity $P_{b}$ should be equal to zero. The minimal SNR, at which the lidar estimate of the dissipation rate by this method still satisfactorily agrees with the assessments of $\varepsilon$ from acoustic anemometer data, is equal to 0.05 [5].

The time-height patterns of the turbulence energy dissipation rate $\varepsilon(h, t)$ were calculated with a height step of $26 \mathrm{~m}$ and a time step of $36 \mathrm{~s}$ from the array of radial velocities $\hat{V}_{r}\left(R_{k}, \theta_{m}, n\right)$ measured with the use of data received for $N=25$ scannings (measurement time is $15 \mathrm{~min}$ ) for each vertical profile of $\varepsilon$. For each height $h$, the SNR values from the array of $\operatorname{SNR}\left(R_{k}, \theta_{m}, n\right)$ estimates were averaged over all the azimuth angles $\theta_{m}$ and over $N=25$ scannings. Then the relative error of the radial velocity $\sigma_{e}$ was calculated by the equation $\sigma_{e}=\left(1+\frac{1}{\mathrm{SNR}}\right) \frac{\Delta v}{\sqrt{N_{a}}}$, where $\Delta v=0.4 \mathrm{~m} / \mathrm{s}$; it
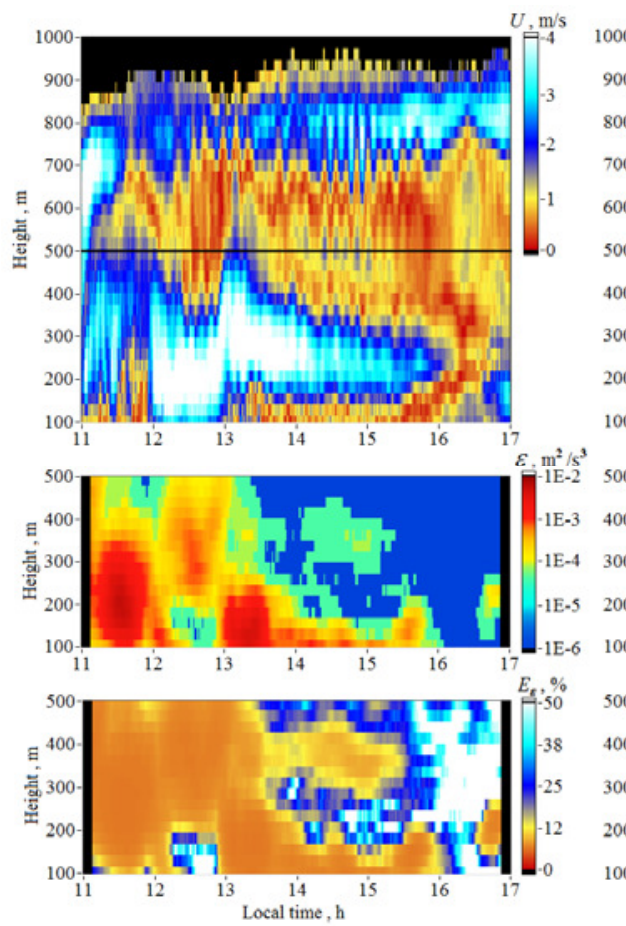

was used for calculation of the relative error of the dissipation rate $E_{\varepsilon}$.

\section{RESULTS}

The Stream Line lidar measurements on August 23, 2015, showed an AIW with a period of 9 min and longitudinal and vertical components of the velocity of about $1 \mathrm{~m} / \mathrm{s}$ and $0.3 \mathrm{~m} / \mathrm{s}$, respectively, which was propagating through the region of experiment for almost $6 \mathrm{~h}$ in the presence of two jet streams. The initial data measured by the lidar from 11:00 to 17:00 on August 23, 2015, satisfied the condition SNR $>0.05$ up to $500 \mathrm{~m}$. We limited to this altitude in the retrieval of the vertical profile of the dissipation rate, while the profiles of the wind velocity components were retrieved up to height of about $900 \mathrm{~m}$.

Figure 1 shows the time-height patterns of the wind speed $U(h, t)$, vertical component of the wind velocity $V_{z}(h, t), \quad \varepsilon(h, t), \quad \operatorname{SNR}(h, t)$, $\sigma_{e}(h, t)$, and $E_{\varepsilon}(h, t)$. The SNR is below a threshold of 0.005 in the black regions.
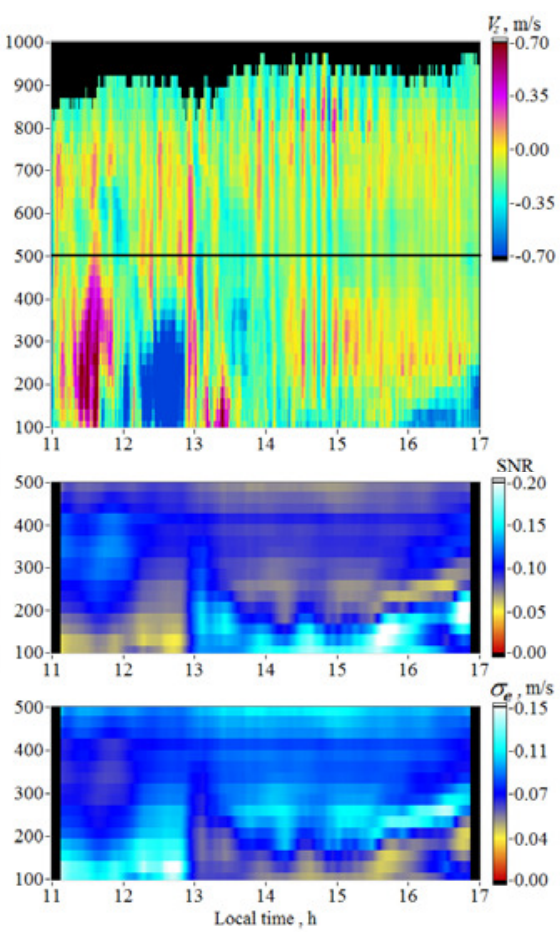

Figure 1 Time-height patterns of the wind speed $U$, vertical component of the wind velocity $V_{z}$, turbulence energy dissipation rate $\varepsilon, S N R$, relative error of the dissipation rate $E_{\varepsilon}$, and the radial velocity error $\sigma_{e}$ retrieved from the Stream Line lidar measurements on the coast of the Lake Baikal on August 23, 2015 
SNR varied from 0.05 go 0.2 in the $100-500 \mathrm{~m}$ layer during that measurement. The radial velocity error does not exceed $0.15 \mathrm{~m} / \mathrm{s}$. The data on the wind speed in Fig. 1 clearly show the presence of two jet streams with speed maxima at altitudes of about 250 and $750 \mathrm{~m}$, respectively (hereinafter, the altitudes are given relative to the lidar location point). The directions of these jet streams are almost perpendicular to each other (the bottom jet stream was directed from north to south through mountains, and the top jet stream was directed from east to west, i.e., from the lake side) [3]. The
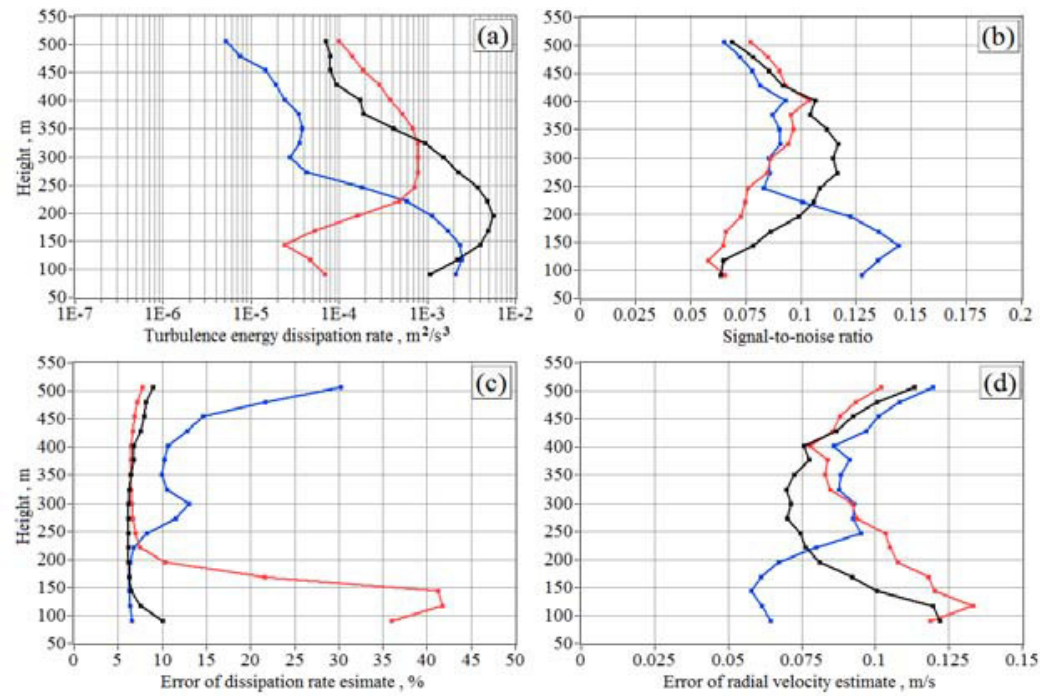

Figure 2 Vertical profiles of the turbulence energy dissipation rate (a), SNR (b), relative error of the dissipation rate estimate (c), and the radial velocity error (d) retrieved from the lidar measurements at 11:30 (black curve),

12:30 (red curve), and 13:30 (blue curve). Data are taken from Figure 1
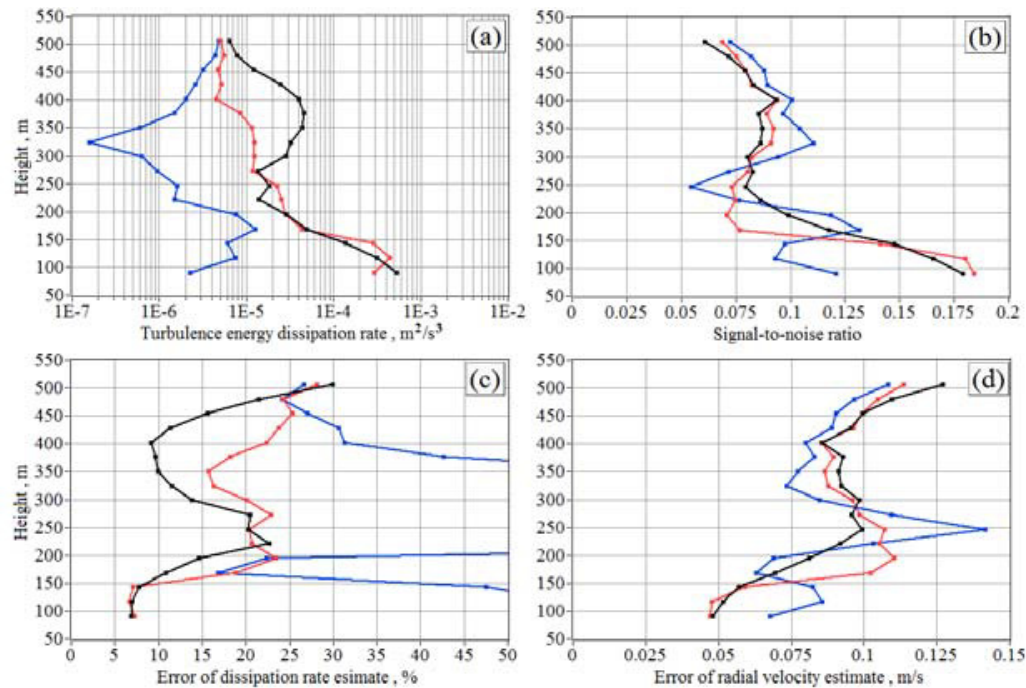

Figure 3 Vertical profiles of the turbulence energy dissipation rate (a), SNR (b), relative error of the dissipation rate estimate (c), and the radial velocity error (d) retrieved from the lidar measurements at 14:30 (black curve), 15:30 (red curve), and 16:30 (blue curve). Data are taken from Figure 1 
Figures 2 and 3 show the vertical profiles of $\varepsilon$, $\mathrm{SNR}, E_{\varepsilon}$, and $\sigma_{e}$ retrieved from Stream Line lidar measurements with an interval of $1 \mathrm{~h}$ (the data are taken from Fig. 1).The turbulence energy dissipation rate obviously can be retrieved from lidar measurements with an error less than $30 \%$ at $\varepsilon \sim 5 \cdot 10^{-6} \mathrm{~m}^{2} / \mathrm{s}^{3}$. The SNR should be no less than 0.075 in this case. The turbulence spatial structure should include an inertial interval with the upper boundary of no less than a half of the longitudinal size of the sounded volume $\Delta z(\Delta z=30 \mathrm{~m}$ for the Stream Line lidar).

\section{CONCLUSIONS}

The results of lidar study of wind turbulence, low level jet streams, and internal atmospheric waves in the boundary layer of atmosphere on the coast of Lake Baikal are presented.

For estimation of the mean wind velocity the modified method of filtered sine wave fitting $[2,3]$ was used. The method modified allows a 1.5 -fold increase in the altitude of retrieval of the mean wind velocity profiles from lidar data as compared to the traditional sine wave fitting.

The method of azimuth structure function [5] was applied for estimation of the turbulent energy dissipation rate from radial velocities measured by the lidar with the use of conical scanning by probing beam around the vertical axis. The validity of this method depends on the magnitude of the dissipation rate measured and the lidar signal to noise ratio. For minimal possible values of the dissipation rate, the method is valid if the signal to noise ratio of lidar echo signal exceeds 0.24.

Few events of the internal atmospheric waves were registered during the experimental campaigns. All the registered AIWs were observed in the presence of low level jet streams. The periods and amplitudes of wave variations of wind velocity during AIWs and parameters of jet streams were determined.
Two dimensional time-height patterns of the wind turbulence dissipation rate during AIW events were obtained as well. It is shown that the wind turbulence in the area occupied by jet streams is very weak. Possibly, it is absent at all, and wind velocity variations in the area of jet streams are caused by mesoscale atmospheric processes. In the process of dissipation of AIWs the wind turbulence strength increases.

\section{ACKNOWLEDGEMENTS}

This study was supported by the Russian Foundation for Basic Research, Project No. 1642-700072.

\section{References}

[1] Banakh, V.A., and Smalikho, I.N., 2013: Coherent Doppler Wind Lidars in a Turbulent Atmosphere, Artech House, Boston and London, 248 pp.

[2] Banakh, V.A., Smalikho, I.N., 2016: Wind sensing in an atmospheric boundary layer by means of micropulse coherent Doppler lidars, Opt. Spectrosc. 121 (1), 1140-1147.

[3] Banakh, V.A., Smalikho, I.N., 2016: Lidar observations of atmospheric internal waves in the boundary layer of atmosphere on the coast of Lake Baikal, Atm. Meas. Tech. 9 (10), 5239 5248.

[4] Kolmogorov, A.N., 1941: Local structure of turbulence in incompressible viscose fluid at very large Reynolds numbers, Doklady $A N$ SSSR 30 (4), 299-303 [in Russian].

[5] Smalikho, I.N., Banakh, V.A., Falits, A.V., Rudi, Yu.A., 2015: Estimation of the turbulent energy dissipation rate from the data measured by the "StreamLine" lidar in the surface layer of atmosphere, Atmos. Ocean. Opt. 28 (10), 901-905 [in Russian]. 